\title{
BDCPS - A Framework for Smart Manufacturing Systems using Blockchain Technology
}

\author{
Raunav Chitkara, John Rajan A
}

\begin{abstract}
Blockchain is going to be the most fundamental technology, and will change the world - going forward. In fact, the revolution has already begun. The birth of Industry

4.0 aka the Fourth Industrial Relution (I4.0), has created aneed for autonomous and integrated, secure manufacturing systems. The current smart systems lack the decentralized decision making and real-time communication infrastructure, which is a condition for adaptive, smart manufacturing systems. In this paper, an autonomous, secure and collaborative platform based on Blockchain technology, is presented to adapt to such results. In support with Internet of Things (IoT) and cloud services, a Blockchain Driven Cyber Physical Production System (BDCPS) architecture is designed to communicate with machines, users, devices, suppliers and other peers.

Using the Smart Contracts feature and trust-less peer-to-peer decentralized ledger feature, BDCPS will validate the claim with a small-scale real-life Blockchain with IoT system. This implementation case study will be running a private Blockchain on a single board computer, and bridged to a microcontroller containing IoT sensors. The applications of this system in automotive manufacturing industry are presented, to proceed towards Industry 4.0.
\end{abstract}

Keywords: Automotive, Blockchain, Industry 4.0, Internet of Things, Manufacturing, Smart Manufacturing

\section{INTRODUCTION}

Blockchain is the distributed ledger technology that is taking the world by storm. It was introduced in 2008, and has created waves in every industry till date.[1] As part of this paper, the research discusses the disruption and impact the technology is making in the manufacturing industry. To understand its use, one must first understand the upcoming trend of I4.0. It is a new transformation that combines traditional manufacturing practices with modern technology. It uses existing techniques and new ones, to create an automated and smart environment. It is the evolution of current production systems using the latest technologies and the internet. The trend is a new revolution using the latest in the digital world to advance manufacturing and enable digital data exchange, along with complete automation. It is based on the fundamental idea of real-time data, autonomous decision making and cyber physical systems. The key idea to I4.0 is smart factories built around smart devices. The building technologies of I4.0 are IoT (Internet of Things) and smart devices. I4.0 is a jump from Industry 3.0, where

Revised Manuscript Received on December 16, 2019

* Correspondence Author

Raunav Chitkara*, SMEC, Vellore Institute of Technology, Vellore, India. E-mail: raunavchitkara@gmail.com

John Rajan A, SMEC, Vellore Institute of Technology, Vellore, India.

E-mail: ajohnrajan@vit.ac.in the data was only monitored and automation carried out. The system is now just more than monitored and controlled - it will be able to communicate, analyze and take decisions on its own. This gives rise to the name Cyber Physical System (CPS).

Blockchain is one of the technological pillars of I4.0. Through this paper, its use in the manufacturing industry is studied - with an implementation case study and examples of its use in the Automotive industry. In moving towards a smart manufacturing system, the major drawback still is the lack of autonomous, decentralized decision making and transparent, secure real-time communication between smart devices, users, suppliers, machines, etc.

This is where blockchain comes in - providing a platform for decentralization of power, autonomous decision making and a secure communication infrastructure for horizontal and vertical integration. Blockchain being secure, decentralized, distributed and through their autonomous Smart Contracts feature, are the perfect answer for this end result.

To do this it will be shown how Blockchain can enhance the automated manufacturing systems by drawing a comparison to existing infrastructures, and provide examples of decentralized decision- making use cases for Blockchain in Automotive Manufacturing Industry.

The aim of the research is to show Blockchain forming the infrastructure for a smart manufacturing. The blockchain technology in collaboration with IoT forms BDCPSBlockchain Driven Cyber Physical Production System. To validate the above claim, a physical small-scale Blockchain with IoT implementation to carry out machine communication and machine maintenance is presented. The implementation case study uses a single board computer running an Ethereum client, and IOT sensors on a microcontroller.

The use cases are endless, especially when the power of IoT is combined with other modern technologies, such as cloud computing, big data and machine learning. But there are other big hurdles that will surface as millions of smart devices will want to secure trustless interactions between themselves and other service providers on the internet. These challenges cannot be met with the current models that are powering IoT interactions, blockchain technology has come as a savior which enable new types of trustless interactions.[2]

Blockchain was introduced as the platform for digital ledger technologies as well as the implementation of digital currencies such as Bitcoin. From there, blockchain has grown into every industry including manufacturing applications. Blockchain holds the key to decentralization of power, and is its biggest advantage.

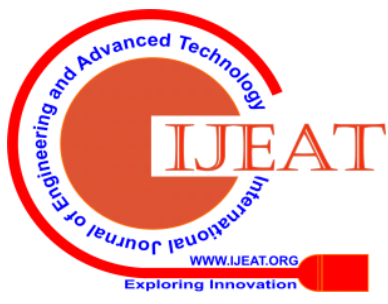




\section{BDCPS - A Framework for Smart Manufacturing Systems using Blockchain Technology}

The users of the blockchain can reach an agreement on a certain activity and register that agreement without the need for a regulatory authority. The activities to be shared could be transactions, records, assets, exchanges, etc. These are secured and shared among all the parties using the blockchain. Blockchain is based on peer-to-peer technology. It uses cryptography to create a secure, transparent distributed shared ledger among the entire group of users - organizations, machines, individuals, products, smart devices, software's, etc. The content added to every block is after being agreed upon by all users, secured and cannot be altered - once appended to the chain. Blockchains allow for creating and tracking an irreversible chain of records, access to live information and measurable components. [3]

\section{RELATED WORK}

\subsection{What is Blockchain} possible to create a digital ledger of any sort of transaction and share that database with all users of the network. It is a type of data structure that can record any transaction; be it exchange of assets, knowledge, data, among the participants of the network. The digital ledger database is shared, replicated and synchronized among all members of the decentralized network. Blockchain is a protocol based on distributed ledger technology, where power is decentralized.

The authority of the blockchain is divided among the participants of the network. And the blockchain is governed by consensus on the additions to the records in the ledger. The blockchain is so named because it is a collection of blocks,

which link together to form a chain. A block contains the data or transaction details for multiple transactions. Every record added in the block has a time stamp and a unique cryptographic signature, thus making the entry validated.

Every ledger is an auditable, unchangeable history of all transactions in the network. The blockchain is secure, tamperevident and permanent. The same copy of the records(ledger) is shared to all member nodes of the network. The blockchain acts as a decentralized authority and doesn't involve any central authority or third- party mediator. The blocks are linked using cryptographic hashing. Member nodes in a blockchain network use a consensus protocol to agree on ledger content, thus making the ledger tamper- evident.[4] The integrity of transactions is maintained by the validation of the records and the block itself using cryptographic hashes and digital signatures.

All the member nodes have the exact copy of ledgers. This makes sure fraudulent transactions are reduced, because to get away with tampering, the same change must be made at many places at exactly the same time - to avoid getting caught. If even one anomaly is found, it has to be checked against all copies of the ledger on all nodes of the network. This is the instrument for decentralization of power.[5]

A hashing technique such as SHA256 algorithm is used.
A blockchain is a peer-to-peer technology that makes it

This ensures that even the smallest alteration to the input will result in a different hash value, indicating changed record. To provide proof that the sender or input party of the transaction are authentic, every node is given a private key - and this is used as a digital signature. The system cannot be single user controlled or undermined unless more than $50 \%$ of the users are unfaithful. The same network protocols apply to all, making the power decentralized, as all participants are equal. Blockchain is built under the assumption that major part of the crowd can be trusted.[6] This is the principle behind decentralization of power.

Each blockchain has to choose a consensus model, which has to be accepted by all. This consensus model will validate and verify the transac-

tions, called mining, and then the system records the transactions

in chronological order - linking them to the previous blocks in the chain. The record cannot be altered or changed unless all users of the blockchain agree to the change. The miners use cryptographic hashing, which is a sort of puzzle solving. The miners mine each block. The miner who solves the puzzle, presents their mined block to the network for verification. Mining requires CPU and energy consumption. The server consumption is used for solving the problem/puzzle. Only after broadcasting the solution to the network will the transaction be added on the blockchain protocol.

\section{COMPONENTS OF A BLOCKCHAIN -}

Miners - People/developers who run the algorithms on servers. Their job is to keep sealing the blocks. Users - People who want to do transactions using the blockchain network. Hash ID

- Transaction ID of the transaction generated by hashing after the mining is completed. If anyone touches the transaction details, the hash ID is changed and can be known by other people. Block

- Collection of multiple records or transactions. Secured and locked with a hash ID as a whole when full. Distributed Ledger

- The same copy of the ledger is distributed to all people in the network to maintain it. Proof of Work (Type of Consensus Model) - The block can be added to the chain only after it has been signed. For this the miner should find the right number (aka Nonce) which leads to the right hash ID. The number is used to seal the block (signed blocks). This is how the transactions in the block are thus verified.

For mining a block or transaction, the miner gets incentive. The incentive could be in the form of cryptocurrency like BitCoin.

Blockchain is very useful in large user networks, as multiple transactions are processed among several users. These transactions are recorded on multiple blocks which are again maintained by thousands of miners across the network

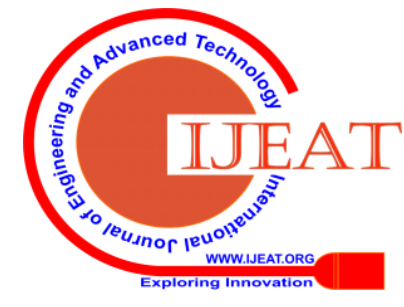


(across the globe) as distributed ledgers. The major question to the skeptical is whether they would trust one authority with all the power, or if they would trust 10000 miners who have nothing to gain from lying.

The first one to calculate the Proof of Work or the Nonce which yields the right Hash ID, gets the incentive (for example BitCoin), for his CPU and Electricity efforts.

Each block is linked to the previous block, and contains the Hash ID of the previous block. If data is tampered with, the Hash ID changes, and this leads to a change in Hash ID of all following blocks. The person will have to mine all the blocks in the blockchain again, which is near to impossible. If someone tries to cheat, it will create a new chain - keeping old one intact. The new chain cannot keep up since all the other system are following the right chain, and he is caught with the anomaly.[6]
Figure 1: Simple structure of blocks of Blockchain

Blockchain is transparent, faster, decentralized, user controlled and cheaper.

Figure 2: Process of Verification

\section{B. Merkle Tree -}

Merkle trees are created by constantly hashing pairs of nodes until there is only one hash left (this hash is called the Root Hash, or the Merkle Root). They are erected from the bottom up, from hashes of individual transactions (known as Transaction IDs).[7]

Figure 3: Merkle Tree Principle

Figure 4: Simplified Bitcoin Blockchain

\section{Link to Previous Block}

\section{Hash of Previous Block}

Nonce

Transaction

Transaction

Transaction

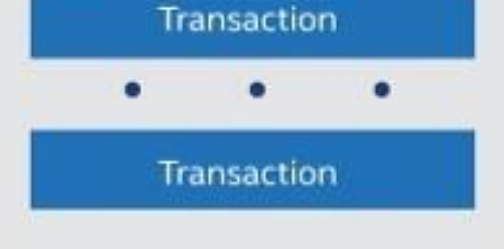

Block N

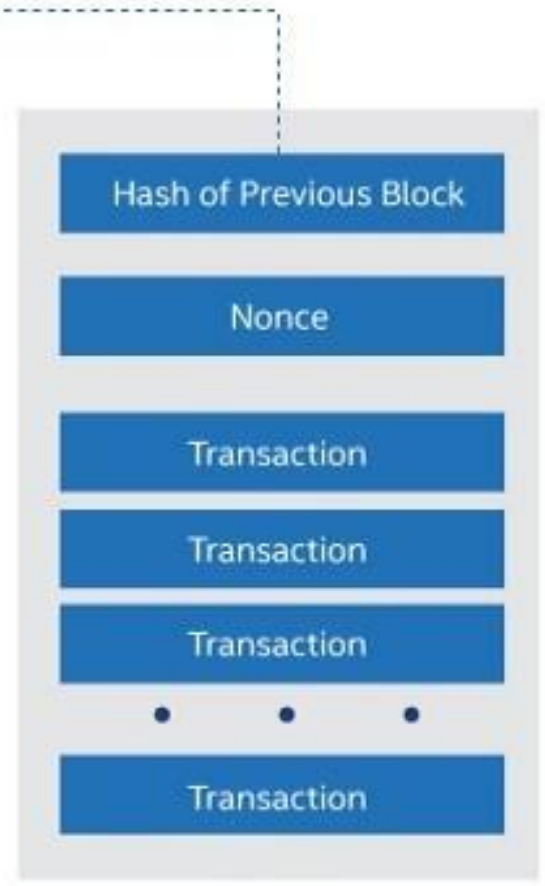

Block $\mathrm{N}+1$

Fig. 1. 
HOW BITCOIN TRANSACTION WORKS

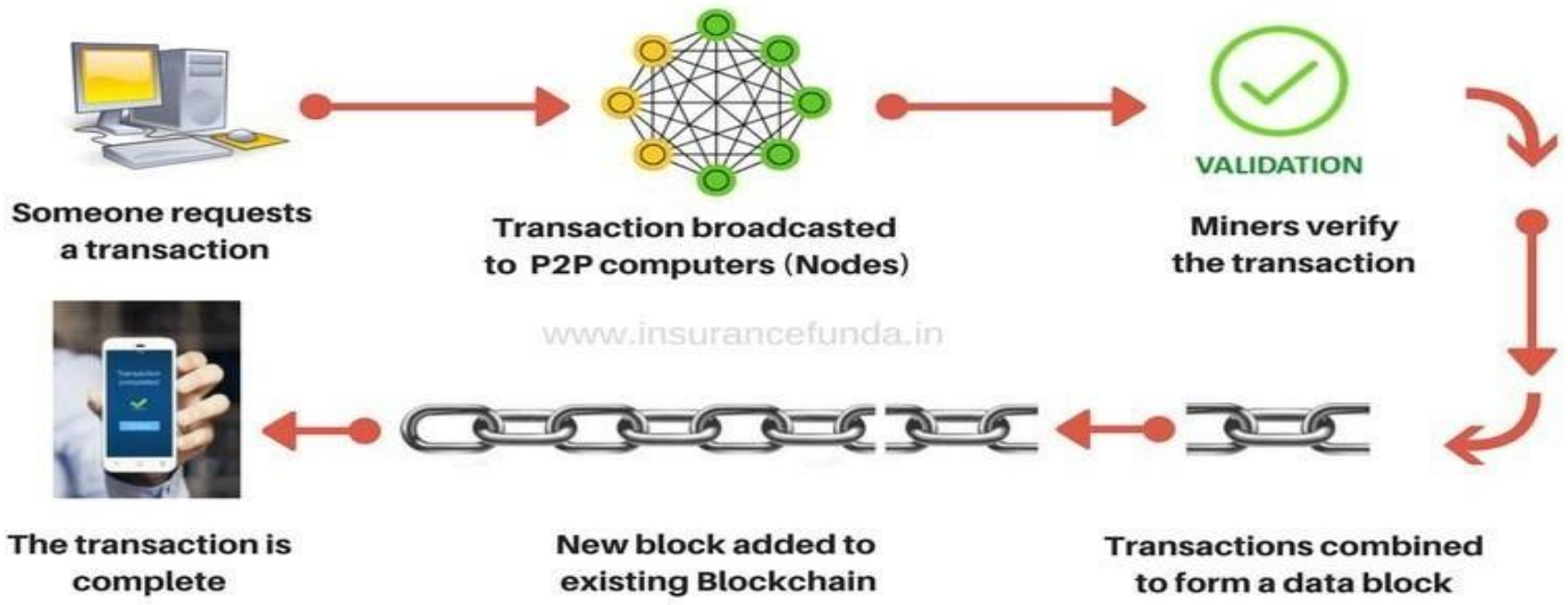

Fig. 2.

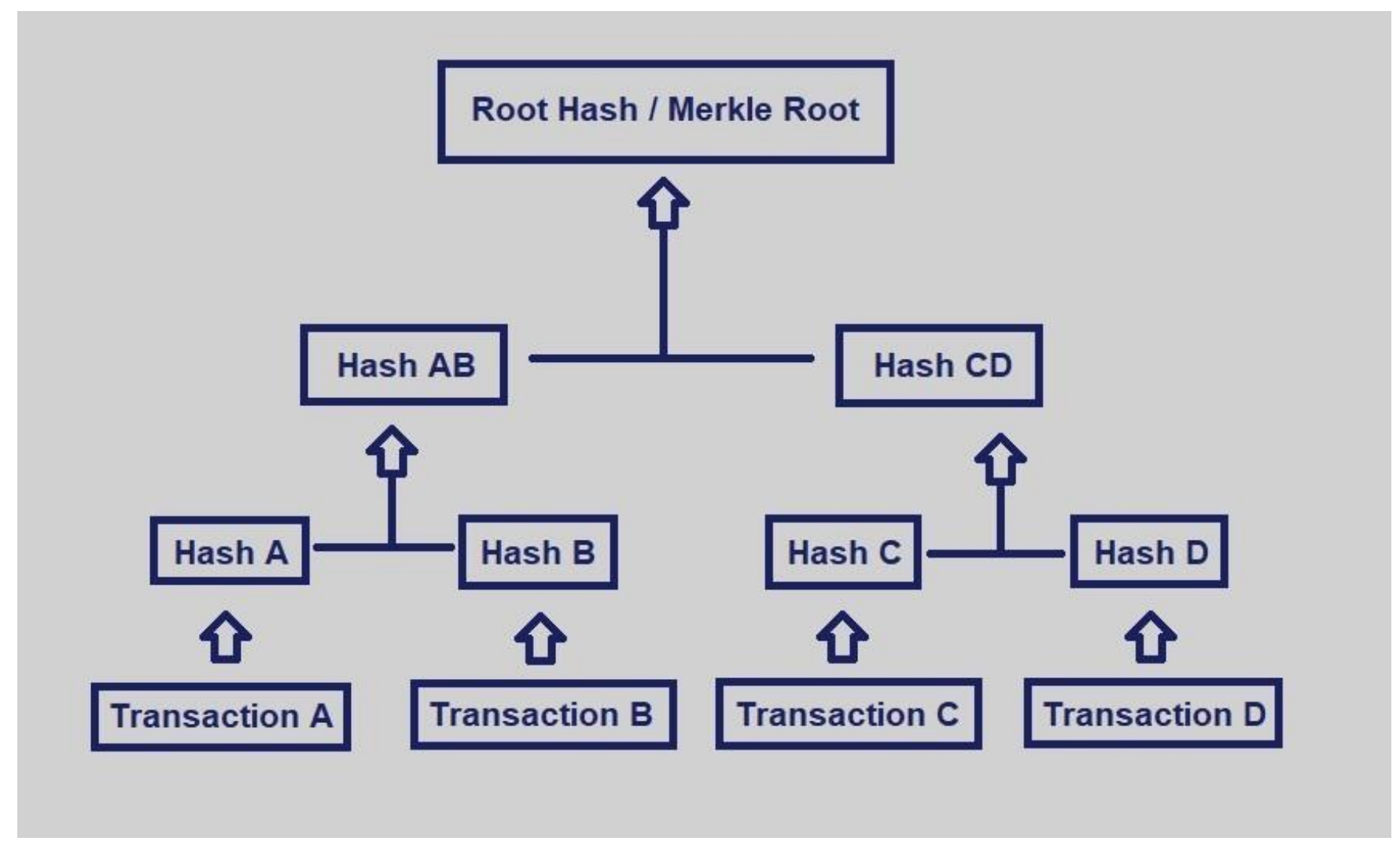

Fig. 3.

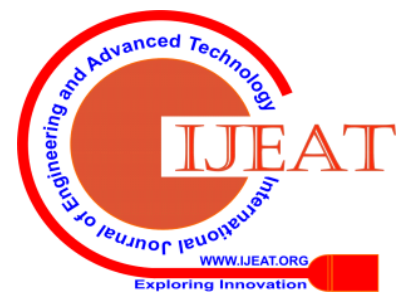




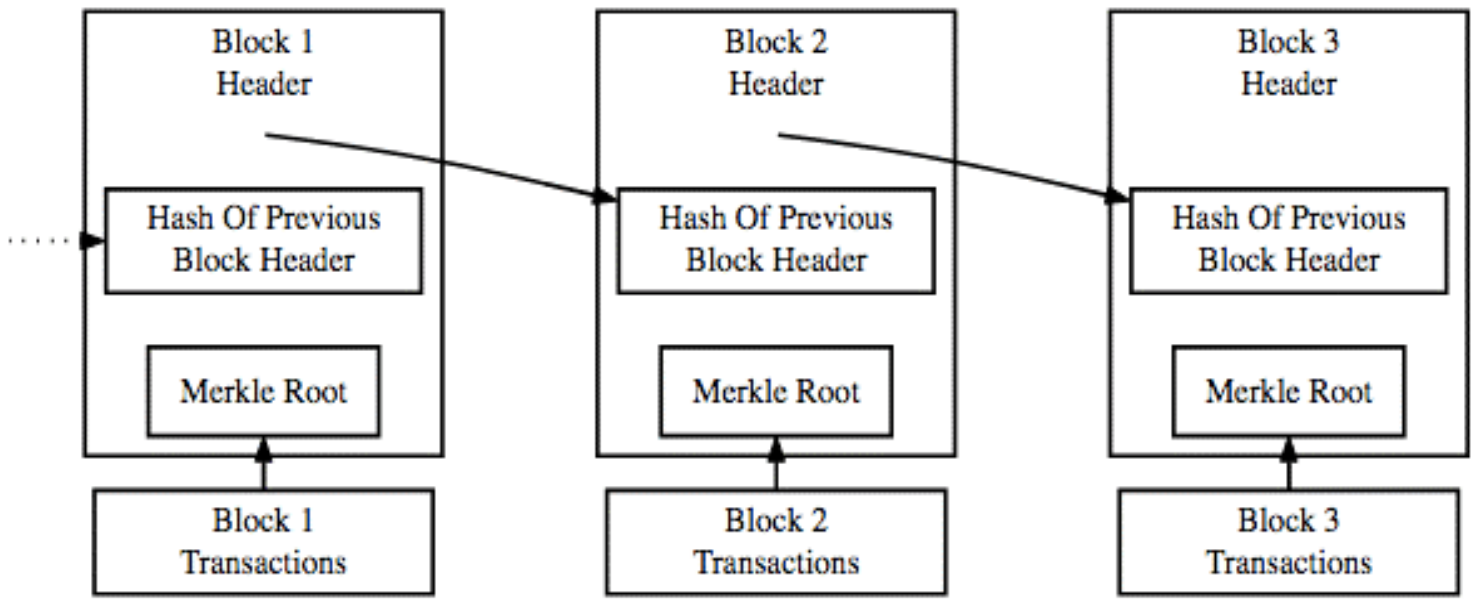

Simplified Bitcoin Block Chain

\section{Signing and Veriftcation of Blocks -}

Figure 5: Signing and Verification

D. Proof of Stake vs Proof of Work -

These are two types of consensus models. Proof of Work works on the premise that every miner has a fair chance of solving for Nonce, and full computer power must be used to mine. Reward goes to first miner to solve the problem. Every miner competes to be the first to find a solution. Proof of Stake works on the

principle that the creator of a new block is chosen in a
Fig. 4.

deterministic way, depending on its wealth or stake (locked up for purpose of validation).[8] The reward for the miners is the transaction fees and there is no block reward. This system is more cost effective. The reward is proportionate to the miners bet on the

block.[9]

Proof of work is extremely resource expensive. And Proof of stake has the drawback of always winning, even on malicious branch chains. This is called Nothing at Stake problem.

\section{common puble kev \\ Digital Signature}
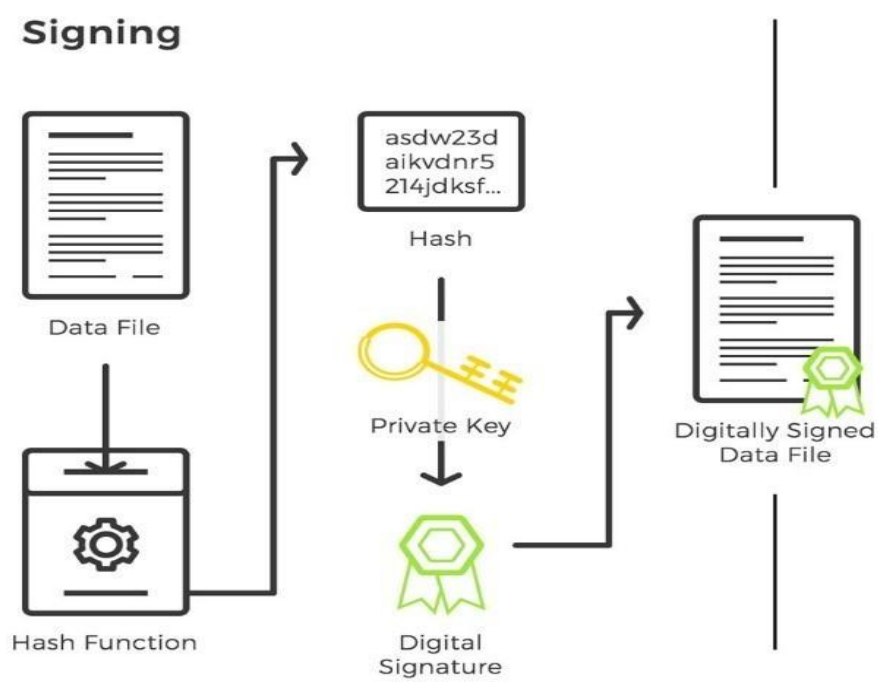

\section{Verification}

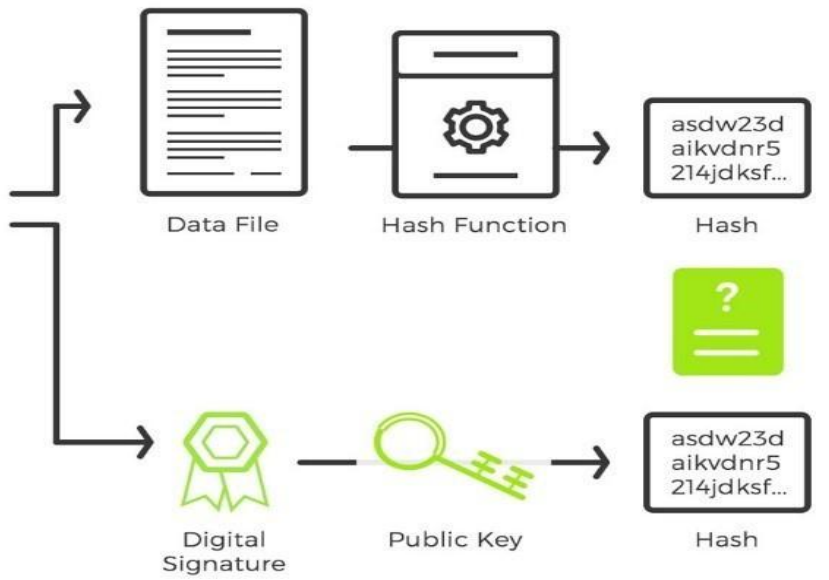

Fig. 5. 


\section{E. Hashing -}

Hashing is a transformation algorithm technique that transforms data of arbitrary size to data of shorter fixed-size or key that represents the original data. Hashing is used to index and retrieve items in a database. Blockchains commonly use SHA256 hashing algorithm. The hash is generated by solving for a number (aka Nonce) that generates a Hash ID that satisfies the general rules set by the network as acceptable. This solving or mining is done by Brute Force method using resources, and incentivized to create takers.[10]

\section{F. Smart Contracts -}

Smart contracts are software programs that exist on a blockchain and form the basis of many of the new blockchain applications and schemes. They are essentially automated dealings that can provide services in exchange for cryptocurrency.

This is actually a computer code running on top of a blockchain containing a set of rules/commands under which the participants to that smart contract agree to interact with each other. If the pre- defined rules are satisfied, the commands automatically enforce. The smart contract code facilitates, verifies and enforces the negotiation or performance of an agreement or transaction. It is a simple form of decentralized automation. A smart contract directly

controls the transfer of data or digital currency or assets between parties or users, under certain conditions. The smart contract defines rules and penalties related to an agreement, and automatically enforces those obligations. These contracts are stored on blockchain technology.[11]

\subsection{Industry 4.0}

Industry 4.0 is a new industrial transformation and revolution that combines traditional manufacturing and industrial practices with modern technology. It is an evolution of current production systems with increased automation and smart technology. It revolves around cyber physical systems - that are mechanisms controlled or monitored by computer-based algorithms, tightly integrated with the internet. This includes robotics, autonomous production and vehicles, machine to machine communication, internet of things, smart factories, wireless sensor devices, artificial intelligence, nanotechnology, quantum computing, biotechnology, additive manufacturing, etc. Industry 4.0 is based on the concept of linking real objects with information processing/virtual objects and processes via information network.

There are eleven major technologies creating the breakthrough for Industry 4.0 -

1. Cloud

2. Cybersecurity

3. IIot -Industrial Internet of Things

4. Horizontal and Vertical systems Integration Simulations

6. Autonomous devices (vehicles, robots, machines)

7. Big data and analyticse

\section{Augmented Reality}

9. Additive Manufacturing

10. Blockchain

\section{Artificial Intelligence}

Industry 4.0 focuses in interconnectivity, automation, machine learning, and real time data. It is a combination of physical production and operations with smart digital technology, machine learning, and big data to create a connected environment that focus on manufacturing and supply chain management. With the fourth industrial revolution the emphasis is on digital technology, with the help of Internet of Things for connectivity. The entire crux is on Cyber Physical sys-tems.[12]

Industry 4.0 will bring with it faster data across machines, real- time simulation, collaborated manufacturing and smarter products. The entire system when changed, will result in higher productivity for businesses. The essence of Industry 4.0 is Internet of Things and Services, and Cyber Physical Systems.

Traditional Manufacturing and Practices+ Latest Technology = I 4.0

The way to execute this latest industrial revolution is through large-scale Machine-to-Machine communication and Internet of Things deployments. Also, producers should move forward to higher automation, better communication and remote monitoring, self-diagnosis, and higher levels of analysis. Factories of the future will be called Smart Factories — freeing up human co-workers for other tasks.[13]

The move is on towards digitization. I4.0 will utilize the IoT and digital physical frameworks, with sensors being able to gather information that can be utilized by makers and providers. Also, the headways in enormous information and amazing examination implies that frameworks can navigate through the gigantic arrangements of information and produce bits of knowledge that can be followed up on quickly. Thirdly, the correspondences framework backing this up is sufficiently secure to be utilized by huge businesses. Another advantage of smart factory is the concept of Virtual production and Digital twins. The digital twin is a digital copy of actual ongoing production line. The virtual copies of the real factory will be linked in real time, and not only a simulation. This availability and use of real-time data will be what leads to higher productivity, cost-reductions and the shift to Industry 4.0 is claimed to create more jobs. [14]

\section{G. Framework of I4.0 [3] -}

- Interoperability - Connecting all manufactur- ing resources to same network as in IoT.

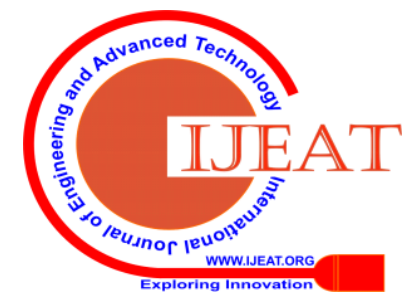




\section{BDCPS - A Framework for Smart Manufacturing Systems using Blockchain Technology}

- Service oriented - All functions and processes offered as services within and outside the company.

- Decentralized

- Real time

- Modular, flexible and adaptive

- Virtualization - Digital twins

\section{H. Technologies needed for I 4.0 -}

Internet of Things: Connections between physical objects like sensors or machines and the internet. In some cases, even the product will have embedded computing. The Industrial IOT will allow communication between these devices and controllers, as necessary. This should result in decentralized analytics and decision making, enabling real-time responses. Industrial IOT is the connections between people, data and machines as they relate to manufacturing.

\section{Smart Factory -}

The concept of Smart Factory is the absolute real-time logical and digital connection of individual production steps, from planning stages to actuators on the shop floor. In the near future, machinery and equipment will be able to improve productivity through self- optimization; systems will autonomously adapt to the traffic profile and network environment. Smart factory demands a shift from independent to interactive, from monitoring to real-time and from stable to dynamic, to take advantage of the value chain of manufacturing and decentralized manufacturing resources.[15]

\subsection{Why Blockchain}

The big drawback of bringing in Blockchain as a standalone technology is that in isolation it cannot create as much of an impact as when combined with other technologies such as Big Data, IOT, Artificial Intelligence and ERP.[16] Blockchain cannot replace existing architecture, but can be used to strengthen and create a new infrastructure. The research gap of Blockchain

being used as the underlying technology to constitute a new manufacturing system is analyzed. Blockchain is the technology which can form the backbone of the system called Blockchain Driven Cyber Physical production system (BDCPS). This is an Industry 4.0 Manufacturing system and Value Chain, in which along with other technologies Blockchain creates the exchange- level infrastructure. The current Manufacturing strategy revolves heavily around enterprise so- lutions like ERP, but with the introduction of Blockchain - these softwares will become decentralized and their dependence will reduce. First off, let us state the key defining features making blockchain the next big thing -

- Near real-time - Updated with every transaction

- Reliable and Available
- Transparent

- Verification and Validation

- Irreversible and Secure

- Immutable

- Digital distributed decentralized ledger validated by network participants without trusted third party

- Smart contracts

- Operation and collaboration across industries

As stated blockchain is the future technological infrastructure for manufacturing systems, and this is because of its transactional record na- ture.[17]

\section{J. Blockchain in the Value Chain:}

At the end of the supply chain, a key differentiator for many companies is the customer's trust in their products quality. Trusted and quality suppliers are carefully checked, selected and managed for every product. In the below infrastructure, Blockchain enables creation and storage of secure digital product memory records - from

Figure 6: Performance classes for Blockchain applications according to [17] .

source of raw material, to their production, including their maintenance and recall history. This is also the case for each part of the product, in- cluding production and quality design. According to the smart Blockchain driven system (BDCPS), with increasing automation and flexible produc- tion processes - blockchain visibility will begin from design files to the manufacturing to the customer purchase, resale and end of life. [18]

Product and assembly ownership, sourcing, authenticity, trading, purchase and warranty use can be protected via blockchains. This would allow multiple supply chain partners to confirm the authenticity of their products. [19]

This smart assembly and Industry 4.0 ready supply chain constitute Blockchain Driven Cyber Physical Production System (BDCPS). The methodology of a web application 


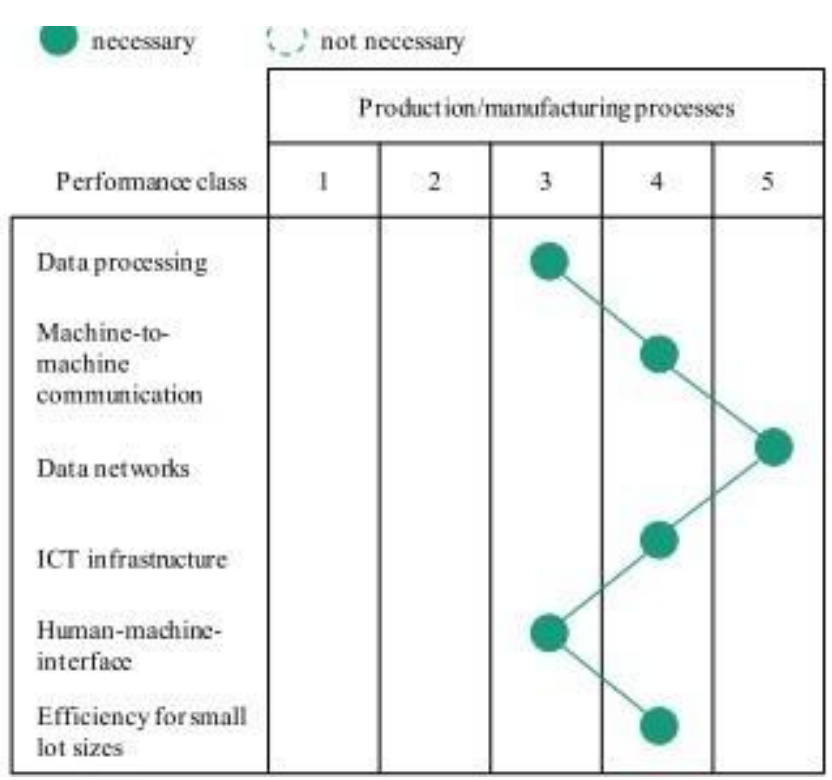

Fig. 6.

based manufacturing would, close to of expanding fabricating profitability and quality, altogether reduce the costs for tracking in enthusiasm of guarantee, support and reusing purposes. Additional investigations about manufacture, version management and recalls would likewise be disentangled. Blockchains would fundamentally streamline forms, particularly those that depend on administrative and quiet submission endorsements.[18]

Trying to achieve the I4.0 vision, blockchain will develop into a new type of manufacturing system in which supply chains will become demand chains and the entire system will become distributed.[28]

\section{K. Smart Manufacturing -}

In combination with IoT, Blockchain can create smart manufacturing devices. Smart manufacturing is nothing but a secure, autonomous and real-time method of manufacturing. It utilizes concepts such as machine communication, distributed manufacturing and smart maintenance to achieve the results of a smart system able to process and produce on its own without human interference. Smart decision making is at the centre of I4.0. Ultimate goal of deploying so many sensors is to achieve autonomous decision making through comprehensive data collection. The realization of smart decision making requires real- time information sharing and collaboration. [20]

\section{METHOD} industry for supply chain optimization. It is useful in supply chain tracking and traceability. But other than supply chain, it really can be used to achieve I4.0 goals in the field of smart manufacturing. Through the extensive research on IoT systems and blockchain technology, one will be able to execute the smart manufacturing ideology as well as an autonomous, decentralized system. [21]

The ideology has been converted into an infrastructure which can be applied for any blockchain with IOT manufacturing
Firstly, blockchain was initially introduced in manufacturing

value chain. Horizon- tal integration is carried out through secure IOT

communication through the blockchain infrastructure machine to machine communication, machine maintenance, etc.

Vertical integration is carried out through communication between the machines and the suppliers as well as up the chain. In horizontal and vertical integration, all data, value and assets are exchanged over the blockchain.[22]

To carry out the results of this research, the methodology of creating an infrastructure and following it up with a case study implementation has been used. This is followed up with the scope and example use cases of this infrastructure in the automotive manufacturing industry.

\section{PROPOSED ARCHITECTURE}

BDCPS revolves around the Industry 4.0 (I4.0) vision. BDCPS is a smart manufacturing production system. It has three key layers -

1. The infrastructure layer - It consists of the machines and the sensors on them. They send real time data from the product or the machine or the part, to the higher level. The infrastructure is mainly made of the Internet of Things technology which speak to each other and nodes, as well as other organizations through the cloud.

2. The Blockchain layer - It consists of the various blockchains running to secure the infrastructure layer as well as provide decision making power. It acts as the communication between the infrastructure IOT layer and the higher enterprise layer. The first type of blockchain is the machine blockchain. This is the medium of communication between the machines as well as between the machines and the cloud, nodes, internet, etc. The machine blockchain is what provides security to the IOT layer and provides the communication platform between all the parties of the manufacturing system. The smart machines would have their own blockchain accounts and the parties/users will be able to communicate with the machines to engage manufacturing and maintenance services. The material blockchain keeps a track of all the parts, and its status. It is useful for part authentication. The various parts and materials are tracked along the supply chain and it holds the chain accountable and traceable. The machine could call for parts directly from the supplier, when short. This relies on the collaborative nature of blockchain - where it is built between all the companies using it. It is the driving force behind distributive and on demand manufacturing. If carried out on a

Figure 7: BDCPS Architecture

global scale, this will be the ideal situation to use a blockchain. The more parties using the global collaborative blockchain - the more secure and efficient it will be. At last, the end-game will be the point at which the innovation itself retreats into the texture of the general assembling activity and we could move into a circumstance where 'supply chains' become 'request chains' and the assembling procedure itself gets disseminated in accordance with the Industry 4.0 vision.

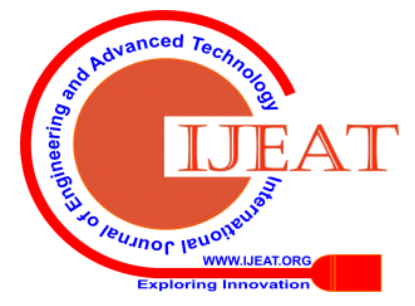




\section{BDCPS - A Framework for Smart Manufacturing Systems using Blockchain Technology}

A blockchain database can enlist the parts utilized in the vehicle fabricating process. Additionally, the assembling procedure itself can likewise be recorded. This could show which people and robots were included at which step, including quality checks. Businesses could eventually take conveyance of each vehicle complete with its own blockchain record.[23]

3. The enterprise layer - This consists of the higher-level applications of BDCPS, such as machine maintenance, autonomous decision making, feeding data to ERP software, 3D printing, supplying data and information to suppliers, etc. These higher- level applications usually utilize enterprise applications - hence the name "Enterprise Layer". This is the most superior layer in the BDPCS architecture. It deals with exchanges and records of assets, data, money and value. This layer will create the biggest impact with relation to agile value chains, faster product innovations, closer customer relationships, and quicker integration with IoT and cloud based services.[24]

\section{CASE STUDY}

Components used - Raspberry Pi(RPi), DHT11 Temp and Humidity sensor, Arduino With Wifi (Microcontroller), Jumper wires, Breadboard

The microcontroller sends the sensor data to the RPi. And the RPi has the blockchain client running, loaded from a laptop or PC on the local network. The IoT sensors, microcontroller, Raspberry Pi and local PC are all connected on the same internet network.

Using a single board computer Raspberry Pi, a microcontroller and some sensors - created a decentralized application (Dapp) for Industrial In- ternet of Things (IIoT) using Blockchain platform Ethereum.

Ethereum - Its an open source Blockchain, which lets users use its clients for connecting to several different blockchains on its network. Ethereum, just like any other blockchain, can be seen as a transaction-based machine where genesis state transitions into a final state by executing transactions incrementally. The final transformation is then considered as the absolute undisputed version

of the state.

Externally owned accounts (EOAs), which are owned and governed by users and have no code associated with them. All transactions on the Ethereum network are initiated by EOAs. These accounts initiate dealings to other EAOs or SC(smart contract) accounts.

Contract accounts are represented by the related agreement code which is put away on the record. Each agreement has an Ether offset related with it. The agreement code's execution is activated by exchanges sent by EOA's or messages sent by different contacts. A transaction is a digitally signed data package that stores a

message to be sent from an externally owned account to another

externally owned account or contract account on the blockchain. Ethereum client used Ether (ETH) as the platform native currency.

Each node in the Ethereum network runs on a Virtual machine, which is an execution environment for smart contracts. Along with Ethereum, BDCPS uses an Ethereum client

Geth, to set up Ethereum on a local machine and communicate with Ethereum network. It can perform tasks such as creating an account and contracts, sending Ether to other accounts, sending transactions to contracts, mining and other tasks related to the Ethereum blockchain.

Using the smart contracts feature, programmed smart contracts were loaded onto the Ethereum test blockchain started with the help of Geth client. To program Smart Contracts, the Solidity IDE with JavaScript language and the Truffle framework were used. The smart contract pro- grammed and migrated to the blockchain is for Machine Maintenance.

This Maintenance smart contract acts as an agreement and can carry out the direct transaction of value (ether in this case) between un- trusted agents - the machine - and the service sellers for scheduling a service when the IoT device identifies the issue with machine.

BDCPS, and this specific usage can be utilized for developing smart diagnostics and self-administration applications for machines where the machines themselves will have the option to screen their state, distinguish issues, and self-sufficiently place administration, or part substitution solicitations to the machine upkeep sellers. Smart contracts among OEM's and sellers for acquirement of provisions and administration of machines can help in mechanizing the machine upkeep errands.[21]

The Python Implementation is created for the Machine connecting to the Blockchain and executing a smart contract under certain conditions. The Python execution of the controller service runs on the IoT device Raspberry Pi. The controller service keeps track of the temperature and humidity levels, and obstruction of unloading of different parts of the machine.

Certain unmistakable standards are characterized to decide whether machine administration solicitation or part substitution request should be set. For example, if the humidity levels of the machine cross a pre-characterized edge for a specific number of times, the controller administration starts a machine administration demand - if the machine is deterred while the humidity is high. This could happen in case of fluid leakage, over- heating, faulty parts, damaged goods, etc.

Figure 8: Flow of Data between IoT and Blockchain

For sending the administration demand, an exchange is sent to the solicitation Service capacity of the Machine Service smart contract between the machine and the service organization.

Following is the Python implementation of a controller service running on the IoT device. It monitors Machine humidity and part proximity to unloading sensor, and sends a service request whenever it reaches

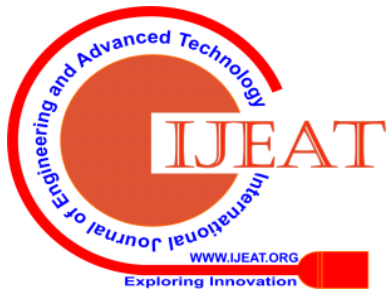


threshold point. [2]

\section{RESULTS}

Ethereum development environment using Ganache CLI was set up. Smart contract for machine service and machine maintenance using the Truffle framework was deployed. To make interaction with the blockchain and contract easier, a simple webpage was used and displayed the machine maintenance status and details. When the machine is not in a good state, the IoT device communicates with the

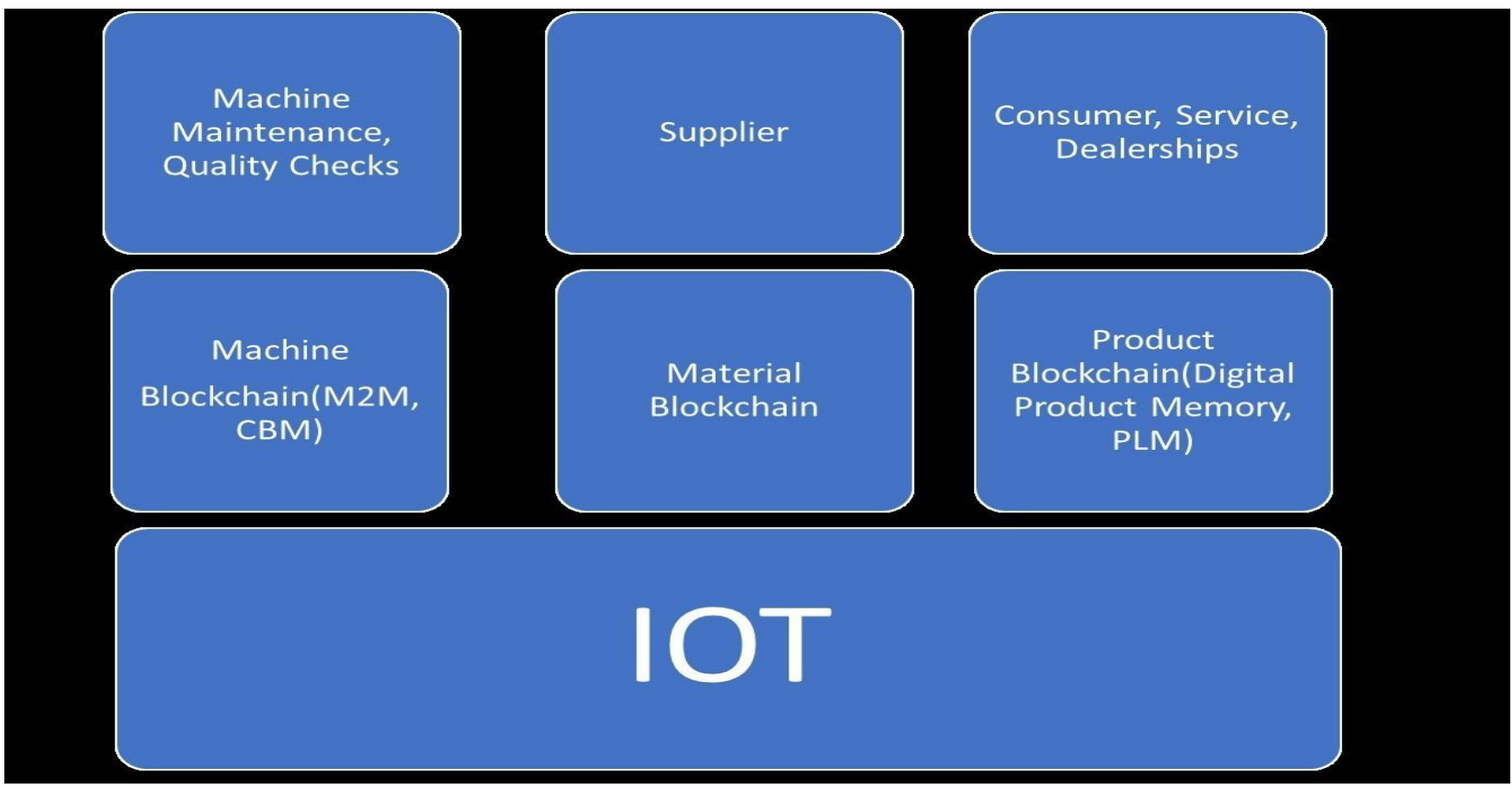

Fig. 7.

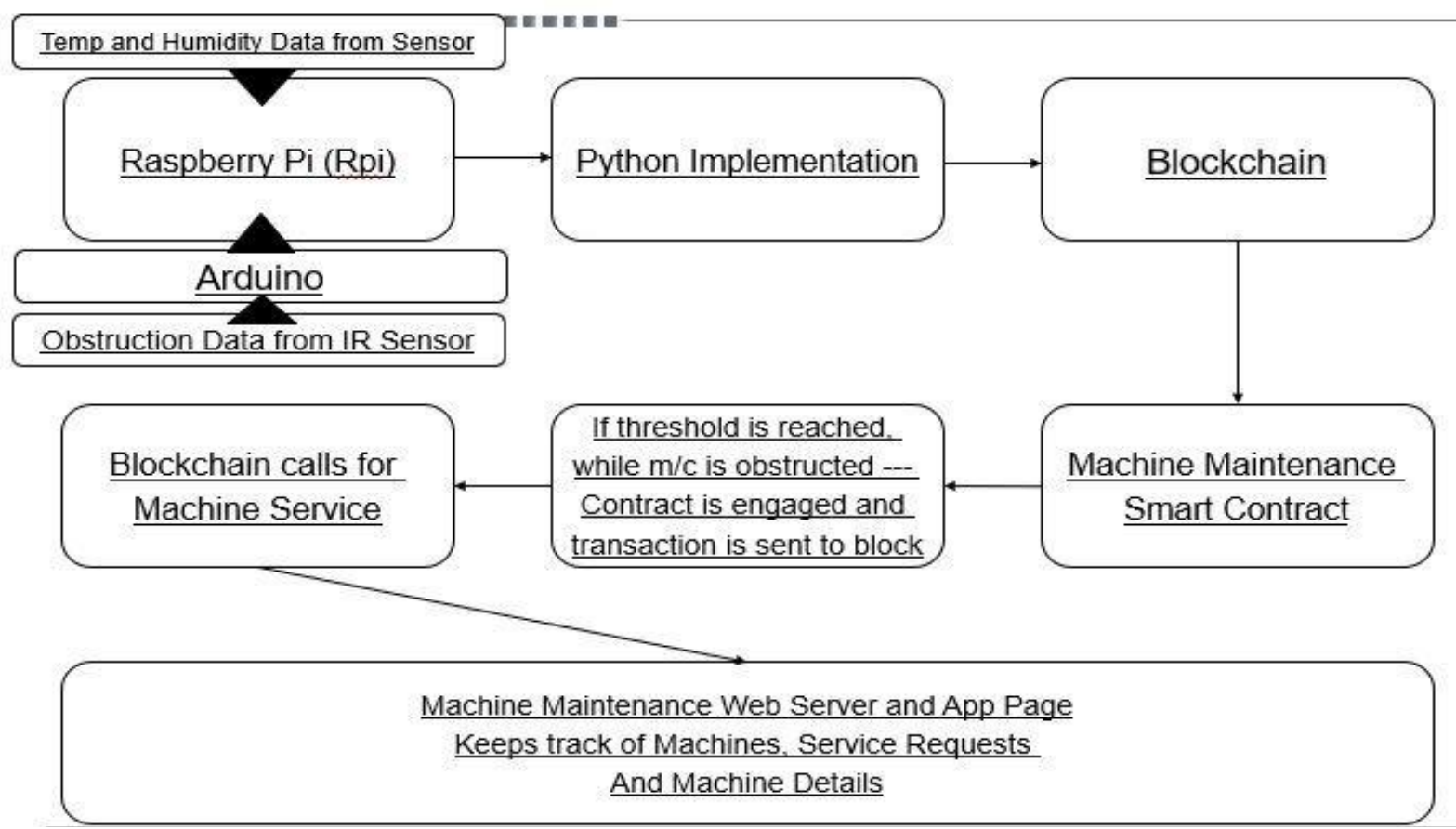

Fig. 8.

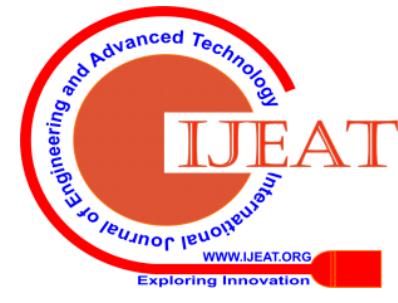




\section{BDCPS - A Framework for Smart Manufacturing Systems using Blockchain Technology}

Blockchain and smart contract via the Python program, and automat- ically calls for machine service to the concerned team. All this is carried out autonomously with the machine node making a

decentralized decision without any human interaction.

\section{DISCUSSION}

Using the implementation given above, the research has proved that the blockchain technology can be used to provide the necessary secure plat- form for IoT communication as well as a decentralized decision-making tool. Thus, BDCPS can be scaled and used for a number of applications. The BDCPS platform is built on the I4.0 vision, and it provides an architecture for smart manufacturing. Toshowcase BDCPS, an implementation was carried out for Machine Maintenance using IoT communication and smart contracts. Each machine has such a setup and is able to make decisions by itself in realtime. The data doesn't have to be sent to a common cloud server for analysis and processing. This brings out the decen-

Figure 9: Code Snippet

tralized nature of blockchain. The decentralized nodes take autonomous decisions and are recorded in a common ledger. The record is unchangeable and secure. The status of each machine can be viewed at a common web page across the entire network. BDCPS architecture is proven to be a secure, trustless and real-time production system. It is integrated, collaborative and transparent in nature. It envisions the I4.0 goals, and works towards and autonomous cyber physical production system. The other important result of BDCPS is that it is easily scalable to take on more IoT devices and more users to the network.

The scope of BDCPS in the automotive manufacturing industry has several dimensions [25] -

1. Distributed manufacturing - Global collaboration of the manufacturing chain among all organizations involved. The parts are manufactured at different factories and brought together only at the end for a final assembly.

2. On demand manufacturing - The machine calls for parts on its own, when required and the entire chain is automated.

3. Machine to Machine communication - Using several such decentralized blockchain nodes, taking pressure of the common cloud server. Machine to machine and consumer to machine transactions with smart contracts and cryptocurrency.

4. Machine service and Part Replacement

5. Machine Provenance and Prognostics

6. Digital Product Life Cycle memory for each product

7. $\quad 3 D$ Printing - The machine calls for parts to be printed immediately as per demand. The blockchain sends the request to a 3D printer, which prints the part and sends it to the machine.

In general, a committed multi-level interconnected blockchain stage dependent on essentials of adaptability and interoperability can profit all - a mutual dispersed record between car producers, vendors, controllers, providers, and so on. This will give higher level of straightforwardness and trust, counteracting questions, and bringing down expenses of upkeep and reviews by effectively following and following. It could likewise streamline forms, ones that depend on administrative and consistence endorsements. [26]

On a concluding note, the challenges of implementing BDCPS are also stated -

1. Companies won't want to share data even with suppliers

2. Might take longer to mine on a smaller network

3. Higher standards for security than actually required

4. Awareness of technology and its benefits

5. Regulation on cryptocurrency is still vague

6. For smaller networks, and few machines - the benefits are almost negligible - works best on large-scale approach

The future scope for research is large and vast. The BDCPS model can form the ideology and methodology behind a scaled- up system - where blockchain forms the fabric of the entire manufacturing system. Further on, it could become a blockchain based system where each machine, user, organization and product are digitally tracked in real-time, with autonomous manufacturing and collaboration beyond distances.

\section{REFERENCES}

1. No Title, (n.d.). https://bitcoin.org/bitcoin .pdf.

2. D. Revan, No Title, (n.d.). https://mediu m.com/@darthrevan344/blockchain-ethereum-io_t-poc-machinemaintenance-part-i-272524c16edf.

3. N. Mohamed, J. Al-jaroodi, Applying Blockchain in Industry 4

4. . 0 Applications, 2019 IEEE 9th Annu. Comput. Commun. Work. Conf. (n.d.) 852-858. doi:10.1109/CCWC.2019.8666558.

5. No Title, (n.d.). https://developer.ibm.co m/tutorials/cl- blockchainbasics-intro-bluemix-trs/.

6. K.D. Jebessa, Decentralization of Power and Local Autonomy in Ethiopian Federal System: A Look at Two Decades Experiment, Http://Www.Sciencepublishinggroup.Com. $\quad 1 \quad$ (2016) 45. doi:10.11648/J.URP.20160103.11.

7. No Title, (n.d.). https://medium.com/@ siddharthram/simplest

8. -explanation-of-blockchain-technology-in-10-minutes-1-2-77cb42c 3eb9c.

9. No Title, (n.d.). https://hackernoon.com/merkle-trees-181cb4bc 30b4.

10. No Title, (n.d.). https://hackernoon.com/consensus-mechanisms

11. -explained-pow-vs-pos-89951c66ae10.

12. No Title, (n.d.). https://coolwallet.io/ethmining/.

13. No Title, (n.d.). https://searchsqlserver.techtarget.com/definiti on/hashing.

14. No Title, (n.d.). https://medium.com/@abhibvp003/smart-cont racts-onthe-blockchain-adeep-dive-in-to-smart-contracts-9616ad2 6428c.

15. No Title, (n.d.). https://en.wikipedia.org/wiki/Cyber-physical_system.

16. W.G. April, 001.Recommendations for implementing the strategic, Acatech. (2013) 4-7. doi:10.13140/RG.2.2.14480.20485.

17. No Title, (n.d.). https://medium.com/air pod-blog/airpod-is- taking-partin-industry-4-0-ee 35ddeadc5f.

18. P. Pinheiro, R. Barbosa, Highlights of Practical Applications of Heterogeneous Multi- Agent Systems. The PAAMS Collection, 430 (2014) 149-160. doi:10.1007/978-3-319-07767-3.

19. Deloitte, Accelerating technology disruption in the automotive market. Blockchain in the automotive industry, (2018). https://www 2.deloi

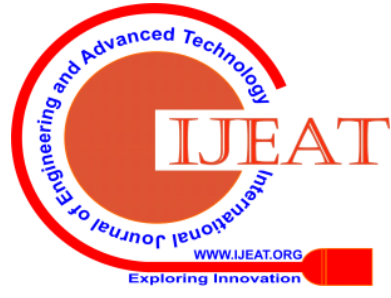


20. tte.com/uk/en/pages/consumer-industrial- products/articles/automotiveblockchain.html.

21. C. Lehmann, C. Schock, R. Technology, Requirements for Blockchain Applications in Manufacturing Small and Medium Sized Enterprises, 24th Int. Conf. Prod. Res. (ICPR 2017). (2017) 155-158.

22. G. Management, M. Consulting, C. White, Blockchain - Technologies

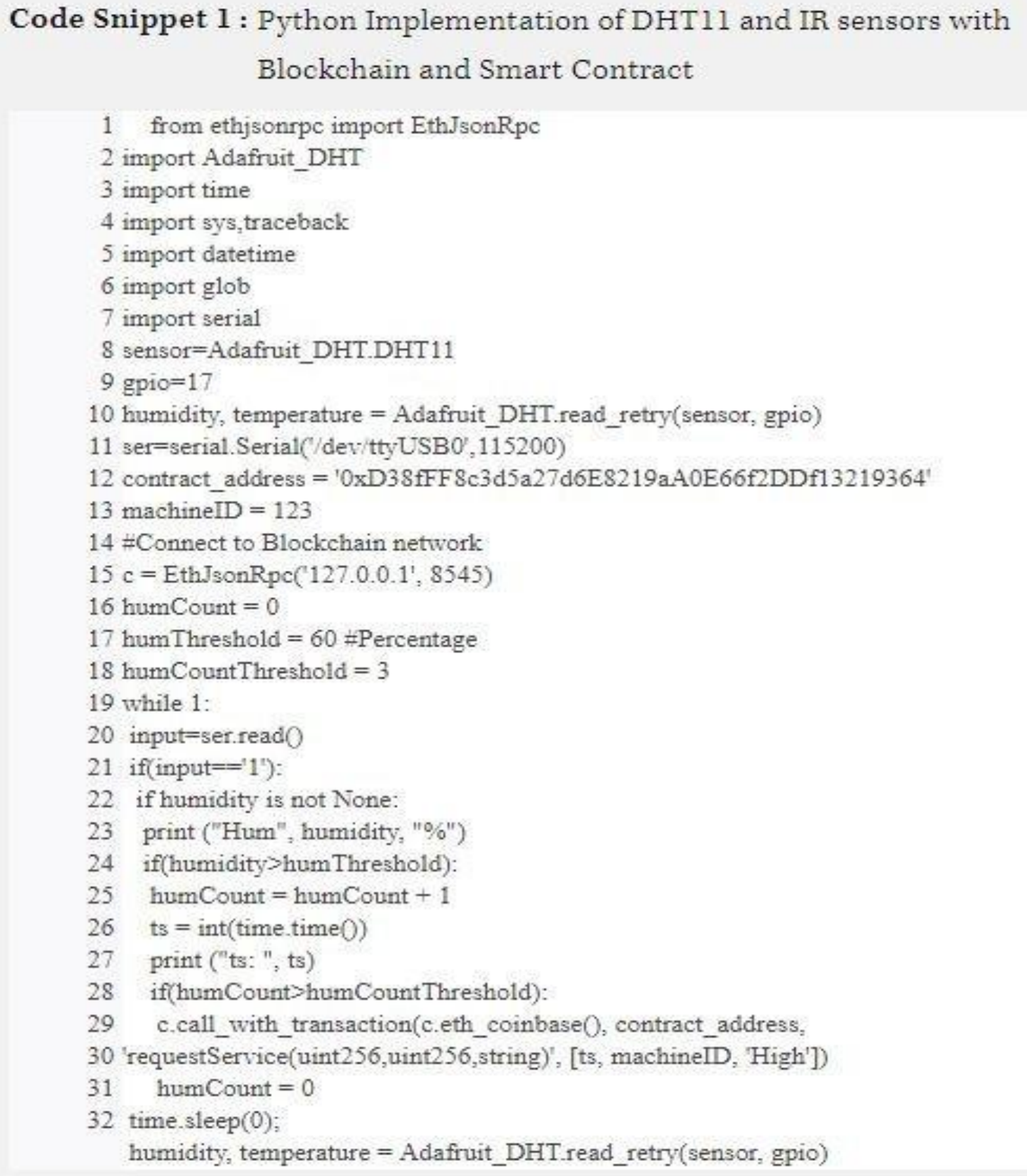

Fig. 9.

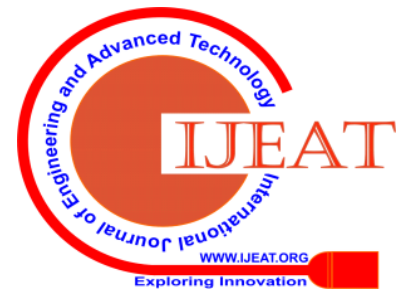


kgo.com/wp-c ontent/uploads/2017/06/WP_Blockchain-online .pdf.

23. How Blockchain Can Slash the Manufacturing "Trust Tax ," (n.d.).

24. P. Zheng, H. Wang, Z. Sang, R.Y. Zhong, Y. Liu, C. Liu, K. Mubarok, S. Yu, X. Xu, Smart manufacturing systems for Industry 4.0: Conceptual

25. framework, scenarios, and future perspectives, High. Educ. SpringerVerlag. 13 (2018) 137-150. doi:10.1007/s11465-018-

26. $0499-5$.

27. A. Bahga, V.K. Madisetti, Blockchain Platform for Industrial Internet of Things, J. Softw. Eng. Appl. 09 (2016) 533-546. doi:10.4236/jsea.2016.910036.

28. M. Marques, C. Agostinho, G. Zacharewicz, R. Jardim- Gonçalves, Decentralized decision support for intelligent manufacturing in Industry 4.0, J. Ambient Intell. Smart Environ. 9 (2017) 299-313. doi:10.3233/AIS-170436.

29. Z. Li, A.V. Barenji, G.Q. Huang, Toward a blockchain cloud manufacturing system as a peer to peer distributed network platform, Robot. Comput. Integr. Manuf. 54 (2018) 133-144. doi:10.1016/j.rcim.2018.05.011.

30. M. Isaja, J. Soldatos, Distributed ledger technology for decentralization of manufacturing processes, 2018 IEEE Ind. Cyber-Physical Syst. (2018) doi:10.1109/ICPHYS.2018.8390792.

31. V. Dieterich, M. Ivanovic, T. Meier, S. Zäpfel, M. Utz,

32. P. Sandner, Application of Blockchain Technology in the Manufacturing Industry, (2017) 1-23. www.twitter.com/fsblo ckchain\%0Awww.facebook.de/fsblockchain.

33. K. Subbiah, B. Ferrarini, J. Maupin, M. Hinojales,

34. S. Kulshrestha, R. Guhathakurta, D. Wright, The Age of Blockchain: A Collection of Articles, (2018) 1-28. doi:10.5281/ZENODO.1202390.

35. N.M. Kumar, P.K. Mallick, Blockchain technology for security issues and challenges in IoT, Procedia Comput. Sci. 132 (2018) 1815-1823. doi:10.1016/j.procs.2018.05.140.

36. G. Halse, What is the future of blockchain in manufacturing?, IT Manuf. (2018). http://www.instrumentation.co.za/9104a.

\section{AUTHORS PROFILE}

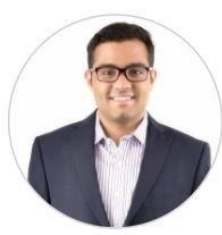

Raunav Chitkara Mechanical Engineer; Automotive, Automation, Cyber Enthusiast

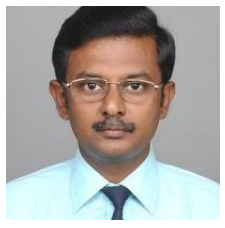

John Rajan A Manufacturing Engineering, Professor, SMEC, Vellore Institute of Technology 